\title{
VIENTO SUR SOBRE LA REGIÓN CÁNTABRO-PIRENAICA OCCIDENTAL (CPO): FRECUENCIA E INFLUENCIA CLIMÁTICA
}

\author{
Kepa San Martín Orbe \\ kepaorbe@gmail.com
}

\begin{abstract}
Resumen: Los flujos de Viento Sur, especialmente durante el periodo otoño-invierno, generan una gran influencia termo-higrométrica (atemperadora y de reducción de la humedad relativa) sobre las condiciones climáticas de la región geográfica conformada en torno al eje central del Golfo de Vizcaya, que se prolonga desde su vertiente peninsular (franja cantábrica oriental) hacia la zona continental (Nueva Aquitania) y ubicada a sotavento del complejo orográfico que estructuran la Cordillera Cantábrica Oriental, los Montes Vascos y losPirineos Occidentales o Atlánticos. El análisis estadístico de los registros diarios de Viento Sur y la correspondencia de estos con los datos respectivos para otras variables climáticas, expresamente para la década de 1961-1971 en la cual se observa un alto cómputo de valores anuales negativos del índice de la Oscilación del Atlántico Norte (NAO), aporta conclusiones muy significativas en cuanto a la afección climática sobre la región de estudio que hemos denominado como Cántabro-Pirenaica Occidental.
\end{abstract}

Palabras clave: Viento Sur, región Cántabro-Pirenaica Occidental, índice NAO.

\section{South winds over the Cantabrian-Western Pyrenean region (CWP): frequency and climate influence}

\begin{abstract}
The South Wind flows, especially during the autumn-winter period, generate a great thermo-hygrometric influence (tempering and reducing relative humidity) on the climatic conditions of the geographical region formed around the central axis of the Bay of Biscay, that extends from its peninsular slope (eastern Cantabrian strip) towards the continental zone (New Aquitaine) and located to the lee of the orographic complex
\end{abstract}

Recibido: 21-03-2021. Aceptado: 05-05-2021. 
that structures the Eastern Cantabrian Mountains, the Basque Mountains and the Western or Atlantic Pyrenees. The statistical analysis of the daily records of South Wind and the correspondence of these with the respective data for other climatic variables, specifically for the decade of 19611971 in which a high count of negative annual values of the index of the North Atlantic Oscillation (NAO) is observed and provides very significant conclusions regarding the climatic effect on the study region that we have called Cantabrian-Western Pyrenees.

Keywords: South Wind, Cantabrian-Western Pyrenean region, NAO index.

\section{Introducción}

En el contexto teórico general de los vientos de montaña y de la influencia de la orografía en el desarrollo y comportamiento termo-dinámico de los flujos de viento (Ledesma, 1959; Gerbier y Berenger, 1961; Hoinka, 1985; Thillet, 1998; Zuñiga y Crespo, 2010) tanto en superficie como en altura, en su estructura vertical como horizontal y a escalas espaciales diferentes, bien planetaria, sinóptica como regional o mesoescalar, ha sido también notablemente estudiado el comportamiento físico y la influencia climática del Viento Sur o vientos del Sur en la interacción de este flujo con la orografía en el ámbito geográfico de la Península Ibérica. Advecciones de Sur, de procedencia oceánica, mayormente atlántica, pero también mediterránea e incluso como flujos perpendiculares a la configuración orográfica impulsados desde el interior peninsular bajo condiciones de inestabilidad atmosférica, bajas presiones invernales sometidas a diferentes procesos dinámicos y gradientes adiabáticos, de enfriamiento $\left(0,6^{\circ} \mathrm{C}\right.$ cada 100 $\mathrm{m}$, en términos generales) y de condensación (perdida de humedad y expansión) en la vertiente de barlovento y de compresión y descenso acelerado y turbulento como flujo catabático (McKnight y Hess, 2000) y subsidente por la vertiente a sotavento, como vientos que se calientan a un ritmo más rápido que el de enfriamiento a barlovento (Pascual, 2013) y de muy baja humedad relativa.

Estas advecciones de sur, generalmente cálidas y húmedas, sometidas a procesos termodinámicos e higrométricos se configuran como vientos de tipo Föhn, flujos de características físicas muy singulares y de gran repercusión climática y medioambiental especialmente sobre la franja a sotavento del prolongado complejo orográfico que componen la Cordillera Cantábrica, los Montes Vascos y los Pirineos Atlánticos u Occidentales (Viers, 1964; Ugarte, 1982; Uriarte, 1996 y Rasilla, 1990, zona peninsular-continental que en nuestro estudio hemos denominado como región Cántabro-Pirenaica Occidental (Fig. 1). 


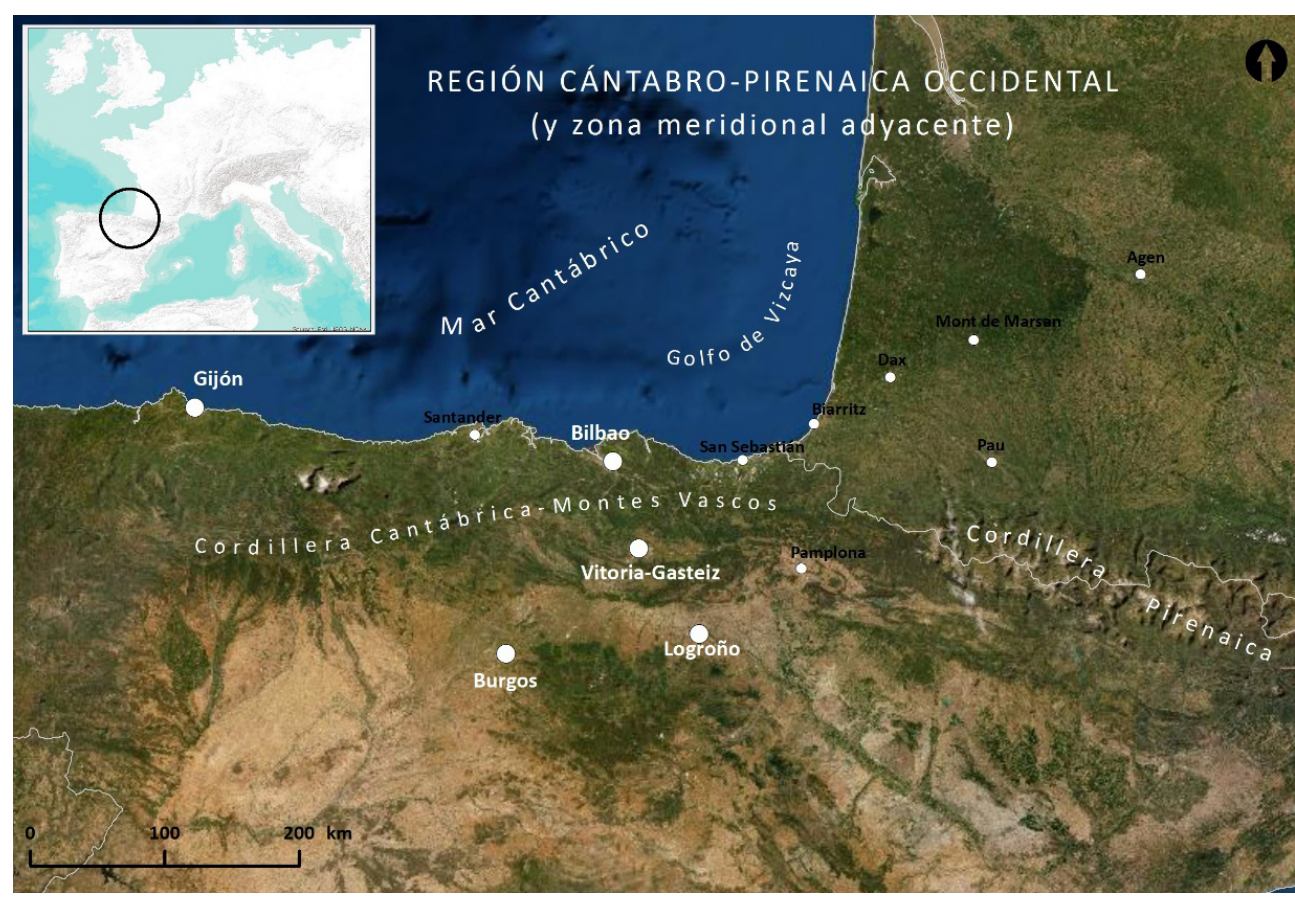

Figura 1. Zona de estudio y estaciones meteorológicas analizadas (puntos blancos gruesos),

Esa notable influencia climática de las advecciones de Sur se produce a lo largo del año; si bien, los vientos del Sur, o Viento Sur, observan sobre la vertiente cantábrica peninsular y áreas de la subregión de los Pirineos Atlánticos franceses, e incluso en otras zonas de la Cuenca del Garona-Adour o región de Nueva-Aquitania, sus más altas frecuencias en el periodo otoño-invierno, entre octubre y marzo (Viers, 1964; Uriarte, 1985; Rasilla, 1990; Fernández García y Rasilla, 1992) y se expresa su influencia con mayor nitidez a través de los parámetros de temperatura y humedad relativa.

El estudio completo que hemos realizado respecto al impacto climático del Viento Sur sobre la región Cántabro-Pirenaica Occidental analiza los datos de Viento Sur registrados en once estaciones meteorológicas de la región (Fig. 1; puntos blancos gruesos) y su correspondencia ${ }^{1}$ con los valores registrados para las variables climáticas que mejor reco-

1. Al hablar de valores o registros correspondientes a una dirección de viento concreta, no pueden ser tomarlos como valores registrados por esa dirección de viento. "Los vientos del norte no registran una temperatura media de $\mathrm{n}^{\circ} \mathrm{C}$, los vientos del norte, en un periodo y localización geográfica concreta, se corresponden con una temperatura media de $\mathrm{n}^{\circ} \mathrm{C}$ ". 
gen y expresan ese impacto de los vientos del Sur. Ahora bien, una primera parte de este estudio, así como este artículo, se centra en el análisis de los registros de Viento Sur (y de otras variables climáticas) expresamente para una seriede once años entre 1961-1971 muy significativa, según la observación aportada personalmente a este estudio por el profesor e investigador del Departamento de Geografía, Urbanismo y Ordenación del Territorio de la Universidad de Cantabria, Dr. Rasilla Álvarez, dado que la misma observa un alto cómputo de valores anuales negativos del índice NAO (Tabla 1) e igualmente para el conjunto de los once años, en contraposición a las décadas finales del siglo XX (19712000) e incluso de un carácter negativo para el total de la serie notablemente más acusado que el correspondiente al de la primera década del siglo XXI (2000-2010).

Tabla 1. Valores (medias) del Índice NAO por décadas (1961-2010) y años de 1961 a 1971 durante el periodo otoño invierno

\begin{tabular}{|c|c|c|c|}
\hline \multicolumn{2}{|c|}{ DÉCADAS } & \multicolumn{2}{|c|}{ AÑos } \\
\hline $1961-1971$ & $-0,17$ & 1961 & 0,012 \\
\hline $1971-1980$ & 0,068 & 1962 & $-0,118$ \\
\hline $1980-1990$ & 0,217 & 1963 & $-0,44$ \\
\hline $1990-2000$ & 0,214 & 1964 & $-0,181$ \\
\hline $2000-2010$ & $-0,042$ & 1965 & $-0,163$ \\
\hline & & 1966 & $-0,136$ \\
\hline & & 1967 & 0,152 \\
\hline & & 1968 & $-0,366$ \\
\hline & & 1969 & $-0,26$ \\
\hline & & 1970 & $-0,31$ \\
\hline & & 1971 & 0,045 \\
\hline
\end{tabular}




\section{2. Área de estudio y metodología}

\section{1. Área de estudio}

La región que hemos definido como Cántabro-Pirenaica Occidental se sitúa en el ámbito geográfico de la Europa suroccidental atlántica, entre las longitudes 5ํ3' 41'” (estación meteorológica de Gijón-Asturias) y 0 37 ' 7" E (estación de Agen-Nueva Aquita-

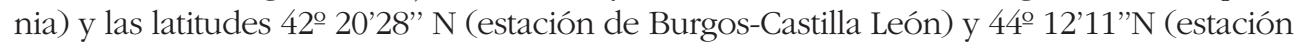
de Agen), analizadas para el total de estudio y que delimitan territorialmente la región. Ahora bien, nos parece oportuno recalcar que aunque el estudio completo se ha realizado para el total de las once estaciones seleccionadas y que aparecen en la Fig. 1, para este artículo en concreto pretendemos incidir en el análisis de los datos registrados en las estaciones seleccionadas para la serie de 1961-1971, periodo con un inusual computo de ocho años, sobre once del total de la serie, en que la media anual del índice NAO fue de signo negativo. Estas estaciones u observatorios son: Bilbao (País Vasco) Indicativo 1082; Gijón (Asturias) Indicativo 1208A; Vitoria (País Vasco) Indicativo 9087; Burgos (Burgos) Indicativo 2331; Logroño (La Rioja) Indicativo 9170.: Todas ellas analizadas a partir de los datos proporcionados por AEMET (Agencia Estatal de Metorología).

Esta ubicación geográfica incluye a la región en el espacio climático de las latitudes medias $\left(35^{\circ}-70^{\circ}\right)$ de la fachada oriental atlántica del Hemisferio Norte, zona en la que las características climáticas, en su sentido más genérico, pero dentro de una amplia diversidad, vienen determinadas por diversos patrones o modelos climáticos. Los procesos de teleconexión entre los diferentes patrones océano-atmosféricos que determinan los tipos de circulación atmosférica y configuración sinóptica en la región del Atlántico Norte-Europa e igualmente son imprescindibles a la hora de analizar la variabilidad climática de continente europeo (ANE-Atlántico Norte-Europa), especialmente de su fachada occidental y por lo tanto el de la región Cántabro-Pirenaica Occidental, situada en la zona suroccidental de esa fachada atlántica continental. El índice de la Oscilación del Atlántico Norte (NAO) e expresa las fluctuaciones entre losvalores de presión registrados en la zona subtropical y subártica del Atlántico Norte (índice NAO). En el Hemisferio Norte, el centro de altas presiones, en las latitudes subtropicales atlánticas, queda fundamentalmente definido por el Anticiclón de las Azores y el centro de bajas presiones en las latitudes altas atlánticas por la Depresión de Islandia (Woollings, T. et al.; 2014).

El índice que expresa esta oscilación se basa en la diferencia de la presión normalizada a nivel del mar (SLP) registradas desde 1864 en Lisboa (Portugal) y Reykjavik (Islandia). Estos valores de presión atmosférica a nivel del mar (SLP) en cada una de estas estaciones se normalizaron obteniendo la media de los valores en ellargo periodo de 1864-1983 y dividiéndola entre la desviación estándar en ese periodo (Hurrell, James \& National Center for Atmospheric Research Staff (Eds.) Last modified 24 Apr 2020. "The Climate Data Guide: Hurrell North Atlantic Oscillation (NAO) Index (station based)." Retrieved from https://climatedataguide.ucar.edu/climate-data/hurrell-north-atlnaticoscillation-nao-index-station-based). 
En el intento de obtener la mayor precisión en la definición del Índice NAO o la diferencia de presión atmosférica respecto ala media estándar estacional entre la Baja de Islandia y la Alta Subtropical, la toma de datos varia en el entorno geográfico de esta última, utilizando también como punto de medición Ponta Delgada, situada en la Isla de Sâo Miguel y capital de las Azores u obteniendo ese índice a través de los datos recogidos en Azores (1865-2002) y en Gibraltar (1821-2007); pero, siempre, en cualquiera de estas mediciones, el otro punto geográfico para la toma de datos de presión, en cuanto a la Baja de Islandia, se sitúa en Reykjavik (Hurrell, Jim. "Índices climáticos NAO/ NAM". Sección de Análisis Climático de CGD)

\subsection{Variables seleccionadas, patrones climáticos y metodología}

El estudio que presentamos se basa en el análisis estadístico de los datos diarios atmosféricos y climáticos registrados y proporcionados por la Agencia Estatal de Meteorología (AEMET) y complementados, en algún caso, desde la base de datos ECA\&D (European Climate Assessment \& Dataset) de cinco estaciones meteorológicas: Burgos, Logroño, Vitoria-Gasteiz, Gijón y Bilbao y para cada periodo otoño-invierno anual (ON-D-E-F-M) de la serie 1961 y 1971.

Dado que la transformación física a que se ven sometidos los vientos del Sur, a medida que avanzan hacia la vertiente de barlovento del sistema orográfico Cántabro-Pirenaico, es fundamentalmente de tipo dinámico, térmico e higrométrico, son los registros medios para el periodo otoño-invierno de las variables o parámetros de Presión atmosférica, Temperatura media y Humedad relativa media (y en menor medida y amplitud territorial la intensidad o fuerza del viento) aquellos que mejor definen, en conjunto y a escala regional, las transformaciones físicas del Viento Sur o vientos del Sur como flujos de montaña sometidos a diferentes procesos adiabáticos de tipo Föhn (enfriamiento, condensación y expansión a barlovento y aceleración, calentamiento, baja humedad relativa y subsidencia a sotavento) y, a su vez, las variables que mejor van a exponer los efectos climáticos de esa transformación en ambas vertientes, pero especialmente entre ellas, septentrional y meridional respecto al conjunto orográfico y entre los diferentes ámbitos territoriales de la región en que se sitúan las estaciones analizadas.

La dirección del viento (D) se ha definido utilizando la rosa de los vientos de ocho sectores u octantes con un arco de circunferencia correspondiente a un ángulo de 45은 cada una. Así, los vientos del noreste (NE) procederían de un sector con el arco de circunferencia correspondiente al ángulo de 22,5 a 67,5; los vientos del este (E) abarcarían un ángulo de $67,5^{\circ}$ a $112,5^{\circ}$, los del sureste de $112,5^{\circ}$ a $157,5^{\circ}$, los de la dirección sur (S) un ángulo de $157,5^{\circ}$ a $202,5^{\circ}$, los del suroeste (SO) un ángulo de 202,5ํa $247,5^{\circ}$, los del oeste $(\mathrm{O})$ se corresponden con un a ángulo de $247,5^{\circ}$ a $292,5^{\circ}$, los del noroeste (NO) de $292,5^{\circ}$ a $337,5^{\circ}$ y los vientos procedentes del sector norte (N) abar- 
carían un arco de circunferencia o sector de dirección correspondiente a un ángulo de $337,5^{\circ}$ a $22,5^{\circ}$.También, tanto para el total de cada serie, como de cada periodo otoñoinvierno, hemos analizado la moda vectorial de cada una de estas ocho direcciones.

Para cada uno de estos parámetros o variables atmosféricas y climáticas se analiza la correspondencia, en cada estación analizada, de sus valores medios para el conjunto de la serie, en el periodo otoño-invierno, con la frecuencia de Viento Sur en ese mismo periodo, expresada tanto en valores porcentuales como absolutos (días o jornadas) y también se determina la dirección sectorial de viento predominante (moda) durante ese periodo en cada estación. La definición o clasificación de las direcciones de viento, se ha realizado tomando como base la rosa de los vientos de ocho direcciones sectoriales agrupadas en cuatro direcciones principales de $135^{\circ}$ respectivamente: Vientos del Norte (NO-N-NE: 292, $5^{\circ}-67,5^{\circ}$ ), Vientos del Este (NE-E-SE: 22, $5^{\circ}-157,5^{\circ}$ ), Viento del Sur (SE-S-SO: 112,5-247,5) y Vientos del Oeste (SO-O-NO: 202,5-337, $5^{\circ}$ ); clasificación que nos resulta más significativa, de mayor interés analítico y más apropiada para un posterior análisis sinóptico que una clasificación en base a la componente del viento, cada una de las cuales consta de un ángulo de $90^{\circ}$.

En cuanto a los registros de presión atmosférica media teniendo en cuenta el ámbito geográfico en que se ubican los observatorios analizados y los tipos más característicos de circulación atmosférica a escala sinóptica en la meteorología de las latitudes medias del Atlántico Norte, hemos establecido dos intervalos de presión o rangos: bajas presiones relativas, $\mathrm{PB}:=<1017 \mathrm{hPa}$ y altas presiones relativas, $\mathrm{PA}:>1017 \mathrm{hPa}$.

Los registros de algunas de las variables o parámetros que hemos analizado (y posteriormente comparado) en cada una de las estaciones u observatorios, han sido clasificadas en intervalos o rangos según el tipo de variable. Este es el caso de la diferenciación entre Bajas presiones (B) y Altas presiones (A) y el de los registros de Temperatura media (T) y Humedad relativa media (H). Esta reducción de todos los registros a un número concreto de rangos para cada variable, nos va a facilitar su análisis, comprensión y significancia estadística, así como el estudio comparativo entre variables. Ahora bien, todas las clasificaciones en rangos o intervalos con las que vamos a realizar el estudio estadístico comparativo se generan tomando como referencia el rango que incluye el valor medio, para el periodo otoño-invernal, correspondiente a cada variable y a partir de este rango se establecen intervalos similares de registros superiores o inferiores al de la media respectiva.

Así, la tabla de Temperatura media (T) para el periodo otoño-invierno se basa en rangos o intervalos de valores definidos a partir del rango que incluye la media otoñoinvernal correspondiente y a partir de ese rango se definen intervalos similares de registros $4^{\circ} \mathrm{C}$ inferiores o superiores al anterior, hasta alcanzar el mínimo y el máximo valor registrado para este parámetro (Tabla 2). 
En el caso del parámetro de Humedad relativa media $(\mathrm{H})$ y dado que la amplitud de valores medios es notablemente menor, los datos respectivos se estructuran en cuatro amplios intervalos, desde el valor mínimo al máximo y en relación al rango o intervalo en el que se sitúa la media. Así, en el caso de la Humedad relativa estos intervalos serían: 0\%-40\%; 40\%-60\%; 60\%-80\% y 80\%-100\%.

El índice de la Oscilación del Atlántico Norte, comúnmente denominado por sus siglas en inglés (NAO), se calcula como la diferencia de las anomalías estandarizadas de presión al nivel del mar, respecto a sus valores promediados en 30 años, entre Azores e Islandia (en términos generales, dado que también se utilizan otras localizaciones de ambos entornos geográficos). Cada valor diario de la serie que vamos a analizar ha sido calculado en relación a la desviación estándar del índice NAO mensual de 1950 a 2000 interpolado al día en cuestión. Para poder ordenar los datos y establecer un análisis de correlación entre ellos, hemos generado una serie del índice NAO, partiendo de los valores diarios del índice (NOAA-CPC), de la misma estructura de periodos trimestrales consecutivos y superpuestos del índice NOI.

Tabla 2. Direcciones sectoriales de viento y rangos o intervalos (primera línea horizontal de celdas) en qué se han clasificado u ordenado por rangos los registros de Temperatura media, a partir del rango que incluye la media otoño-invernal

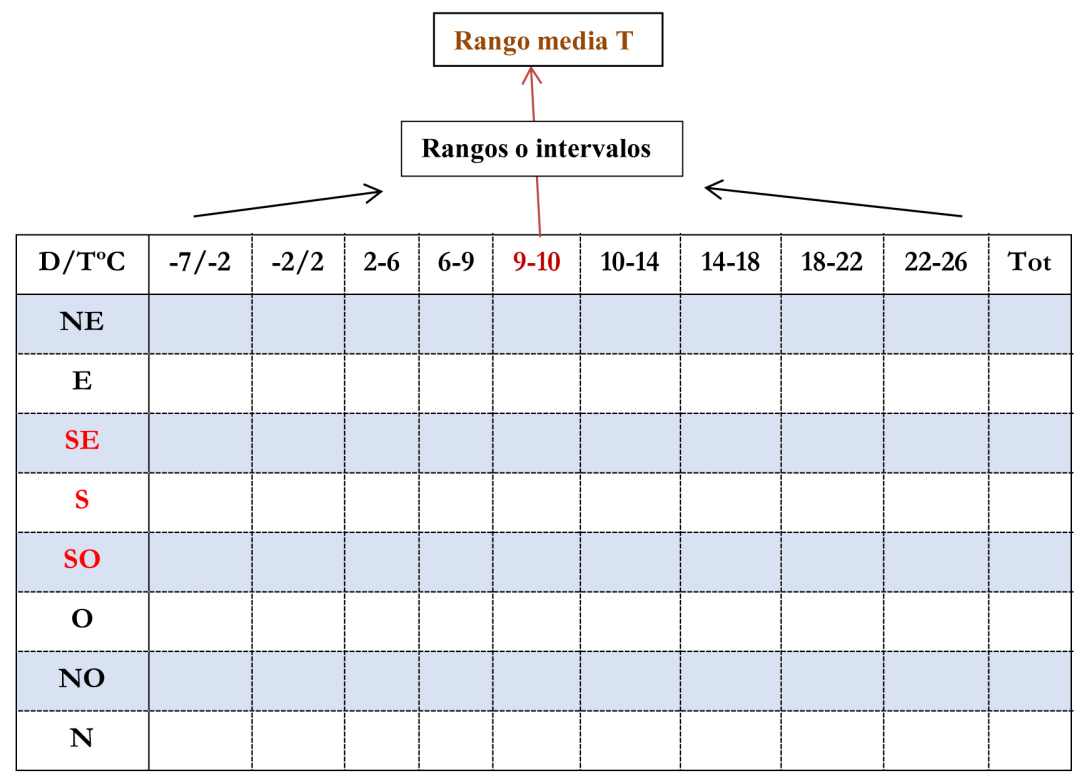




\section{Resultados}

\subsection{Frecuencia otoño-invernal de Viento Sur (Fr \%)}

La frecuencia otoño-invernal de Viento Sur (VS), o valor porcentual de jornadas otoño-invernales en las que es predominante el viento procedente de algunas de las direcciones sectoriales de Sur (SE-S-SO), es muy superior en las estaciones situadas en la vertiente septentrional o a sotavento del sistema orográfico Cántabro-Pirenaico Occidental peninsular, estaciones de Bilbao, 54\% y Gijón, 51\%. En estas dos estaciones y durante el periodo otoño-invierno son predominantes las situaciones del sureste (SE), mientras los vientos del suroeste (SO) son predominantes en la estación de Burgos, pero en esta estación la frecuencia otoño-invernal de Vientos Sur (VS) es muy inferior, 39\%. De entre las jornadas o "días de Sur" (VS) la dirección sectorial sureste (SE) es la que mayor frecuencia observa en las estaciones de Bilbao, Gijón y Logroño y la dirección suroeste (SO) en las de Vitoria y Burgos (Tabla 3).

Tabla 3. Dirección de viento predominante para el periodo otoño-invierno (OI) en cada estación de la región analizada; frecuencia conjunta de Viento Sur (VS) y frecuencia (Fr\%) de jornadas con vientos predominantes del cada una de las tres direcciones sectoriales de Sur (SE-S-SO)

\begin{tabular}{|l|c|c|c|c|c|}
\hline \multicolumn{1}{|c|}{ Fr\% } & OI & VS & SE & S & SO \\
\hline Bilbao & SE & 54 & $\mathbf{2 0}$ & 17 & 17 \\
\hline Gijón & SE & 51 & $\mathbf{1 9}$ & 17 & 15 \\
\hline Vitoria & N & 27 & 5 & 5 & 17 \\
\hline Burgos & SO & 39 & 12 & 6 & $\mathbf{2 1}$ \\
\hline Logroño & NO & 23 & $\mathbf{1 8}$ & 3 & 2 \\
\hline
\end{tabular}

Los vientos del Sur (SE-S-SO), como dirección de viento principal o cardinal, observan conjuntamente y para el periodo otoño-invierno, la frecuencia más alta, en comparación al resto de direcciones cardinales; Vientos del Este (NE-E-SE), Vientos del Oeste (SO-O-NO) y Vientos del Norte (NO-N-NE) y esto es así especialmente en las estaciones de Bilbao y de Gijón, si bien en esta última con un valor de frecuencia porcentual muy ligeramente superior a la de los Vientos del Este (VE). En la estación de Vitoria predominan los Vientos del Oeste (VO) y del Norte (VN), ambos con igual valor porcentual de frecuencia; en Burgos los Vientos del Este (VE) y los Vientos del Oeste (VO) en la estación de Logroño (Tabla 4). 
Tabla 4. Frecuencia otoño-invernal (Fr \%) correspondiente a cada dirección cardinal o principal de viento (VE, VS, VO, VN) en cada una de las estaciones meteorológicas de la región analizadas

\begin{tabular}{|c|c|c|c|c|c|}
\hline Fr \% & Bi & Gi & Vi & Bu & Lo \\
\hline VE & 42 & 50 & 7 & 47 & 27 \\
\hline VS & 54 & 51 & 27 & 39 & 23 \\
\hline VO & 31 & 26 & 39 & 29 & 60 \\
\hline VN & 24 & 22 & 39 & 27 & 46 \\
\hline
\end{tabular}

\subsection{Temperatura media (T) y Viento Sur (VS)}

La temperatura media ( $\mathrm{T}$ ) para el periodo otoño-invierno en las estaciones a sotavento del Sistema Cántabro-Pirenaico Occidental es notablemente superior; Bilbao y Gijón, $11^{\circ} \mathrm{C}$ en ambos casos, a la que registran las estaciones de Vitoria, $7^{\circ} \mathrm{C}$; Burgos, 5ำ y Logroño, $9^{\circ} \mathrm{C}$. Esta tendencia es muy similar, en lo que a los datos de Temperatura media (T) se refiere, para el conjunto de jornadas o días otoño-invernales de Viento Sur (OI); si bien esa media es superior a la del periodo otoño-invierno en Bilbao, $12^{\circ} \mathrm{C}$, en Vitoria, 9ํㅡ y en Burgos, 6ำ (Tabla 7).

Se observa una notable transición positiva en los datos otoño-invernales de temperatura media (T), igualmente en los correspondientes al conjunto de situaciones de Sur (VS) y a cada una de las direcciones sectoriales de Sur (SE-S-SO) entre la estación de Burgos y la de Vitoria, del orden de entre $3^{\circ} \mathrm{C}$ y $5^{\circ} \mathrm{C}$ y de esta última hacia las estaciones septentrionales de Gijón y Bilbao, del orden de entre $2^{\circ} \mathrm{C}$ y $3^{\circ} \mathrm{C}$. De tal forma que, para situaciones de Viento Sur (VS) la diferencia en temperatura media (T) entre Burgos y Gijón es de $5^{\circ} \mathrm{C}$ y de $6^{\circ} \mathrm{C}$ entre Burgos y Bilbao, legando a ser de $7^{\circ} \mathrm{C}$ entre estas dos últimas estaciones para flujos del sureste (SE) (Tabla 5).

En el mismo sentido, esa transición en valores de Temperatura media (T) otoño-invernal y para el conjunto de jornadas de Viento Sur (VS), en cualquiera de sus direcciones sectoriales, se observa entre la estación de Burgos y la de Logroño, con un incremento térmico del orden de entre $3^{\circ} \mathrm{C}$ y $4^{\circ} \mathrm{C}$; Burgos y Vitoria de entre $3^{\circ} \mathrm{C}$ y $5^{\circ} \mathrm{C}$, de $3^{\circ} \mathrm{C}$ entre Vitoria y Bilbao para vientos de la dirección sectorial sur (S) e igualmente de $3^{\circ} \mathrm{C}$ entre Logroño y las estaciones de Gijón y Bilbao para flujos del sureste (SE) (Tabla 5). 
Tabla 5. Valores de temperatura media $\left(\mathrm{T}^{\circ} \mathrm{C}\right)$ para el periodo otoño-invierno, conjunto de situaciones de Viento Sur (VS) y conjunto de jornadas con vientos predominantes de alguna de las direcciones de sur (SE-S-SO), en cada una de las estaciones de la región analizadas

\begin{tabular}{|l|c|c|c|c|c|}
\hline \multicolumn{1}{|c|}{ T } & OI & VS & $S E$ & $S$ & $S O$ \\
\hline Bilbao & 11 & 12 & 12 & 12 & 11 \\
\hline Gijón & 11 & 11 & 12 & 11 & 11 \\
\hline Vitoria & 7 & 9 & 10 & 9 & 9 \\
\hline Burgos & 5 & $\mathbf{6}$ & 5 & $\mathbf{6}$ & $\mathbf{6}$ \\
\hline Logroño & 9 & 9 & 9 & $\mathbf{1 0}$ & 9 \\
\hline
\end{tabular}

Las jornadas otoño-invernales con vientos predominantes del Sur o Viento Sur (VS), en cualquiera de sus tres direcciones sectoriales (SE-S-SO), registran de media y conjuntamente una Temperatura media (T) superior al conjunto de jornadas con vientos de las otras tres direcciones cardinales; vientos del Este (VE), vientos del Oeste (VO) y vientos del Norte o Viento Norte (VN), en las estaciones de Bilbao, Vitoria y Burgos. Estos registros correspondientes al conjunto de jornadas de Viento Sur (VS) en esas tres estaciones son entre $1^{\circ} \mathrm{C}$ y $3^{\circ} \mathrm{C}$ superiores a los registros de Temperatura media cOrrespondientes al conjunto de jornadas con vientos predominantes de las otras direcciones cardinales (VE, VO, VN) Mientras, en la estación de Gijón la temperatura media más alta corresponde al conjunto de jornadas con vientos del Este (VE) y del Norte $(\mathrm{VN})^{2}$ y en la estación de Logroño las cuatro direcciones cardinales de viento observan el mismo valor de temperatura media (Tabla 6).

Tabla 6. Valores de temperatura media (T) otoño-invernal correspondientes a cada dirección cardinal de viento (VE, VS, VO, VN), en cada estación analizada

\begin{tabular}{|c|c|c|c|c|c|}
\hline $\mathbf{T}$ & $\mathbf{B i}$ & $\mathbf{G i}$ & $\mathbf{V i}$ & $\mathbf{B u}$ & Lo \\
\hline $\mathbf{V E}$ & 11 & 12 & 8 & 5 & 9 \\
\hline $\mathbf{V S}$ & 12 & $\mathbf{1 1}$ & $\mathbf{9}$ & $\mathbf{6}$ & $\mathbf{9}$ \\
\hline $\mathbf{V O}$ & 11 & 11 & 7 & 5 & 9 \\
\hdashline $\mathbf{V N}$ & 9 & 12 & 6 & 4 & 9 \\
\hline
\end{tabular}

2. Estos vientos del Norte en Gijón, se desplazan muy cercanos angularmente por el límite inferior de los Vientos de Este (VE). 


\begin{tabular}{|c|c|c|c|c|c|c|c|c|c|c|c|}
\hline & \multicolumn{5}{|c|}{$>$ rango media $\mathrm{T}(\mathrm{OI})$} & & \multicolumn{5}{|c|}{$>$ rango media $\mathrm{T}(\mathrm{OI})$} \\
\hline$\% \mathrm{D}^{3}$ & $\mathrm{Bi}$ & Gi & Vi & $\mathrm{Bu}$ & Lo & \%Tot & $\mathrm{Bi}$ & Gi & Vi & $\mathrm{Bu}$ & Lo \\
\hline VE & 39 & 38 & 59 & 43 & 41 & VE & 47 & 50 & 11 & 42 & 32 \\
\hline VS & 45 & 37 & 59 & 55 & 44 & VS & 66 & 51 & 39 & 44 & 28 \\
\hline VO & 34 & 33 & 47 & 57 & 29 & VO & 25 & 23 & 44 & 34 & 51 \\
\hline VN & 20 & 44 & 31 & 37 & 31 & $\mathbf{V N}$ & 14 & 25 & 30 & 20 & 41 \\
\hline$M(O I)$ & 11 & 11 & 7 & 5 & 9 & $M(O I)$ & 11 & 11 & 7 & 5 & 9 \\
\hline
\end{tabular}

Tabla 7. Número de jornadas, en valor porcentual (\%D), para cada dirección principal o cardinal de viento [vientos del Este (VE); vientos del Sur o Viento Sur (VS); vientos del Oeste (VO) y en cada estación meteorológica de la región analizada, en que se registran valores de temperatura media superiores ${ }^{4}$ al rango de la media respectiva para el periodo otoño-invierno [\% $\mathbf{D}>$ rango media $\mathbf{T}$ (OI)]. Valor porcentual que ese número de jornadas, \%D, representa sobre el total de jornadas o días otoño-invernales en los que se registran valores de temperatura media superiores al rango de la media respectiva para el periodo otoño-invernal [\%Tot. > rango media $\mathbf{T}(\mathbf{O I})$ ]. Temperatura media (T) otoño-invernal correspondiente a cada estación de la región analizada. M (OI): Temperatura media periodo otoño-invierno

3. Los valores porcentuales (\%D) para cada dirección cardinal o principal de viento (VE-VS-VO-VN) no se obtienen de la media entre los valores porcentuales correspondientes a cada dirección sectorial con registros superiores al rango de la media otoño-invernal y respecto a sus jornadas otoño-invernales respectivas, sino de la relación entre el conjunto de jornadas con vientos predominantes de cada una de las dirección cardinales que observan registros superiores al rango de la media otoño-invernal, en relación al total de las jornadas otoñoinvernales en que cada una de esas direcciones cardinales de viento es la dirección predominante.

4. Valores superiores al rango... es igual a decir: valores o registros incluidos en los rangos superiores al rango...; pero, la primera expresión hace más ligera la redacción. 
En todas las estaciones analizadas, excepto en la de Gijón, más del 43\% de las jornadas otoño-invernales con vientos predominantes del Sur (VS) registran valores de temperatura media (T) superiores al rango que incluye su media otoño-invernal respectiva y estos valores porcentuales superan el $54 \%$ en las estaciones de Vitoria y de Burgos; si bien, son valores superiores respecto a rangos en que se incluyen sus medias otoñoinvernales notablemente más bajos; $7^{\circ} \mathrm{C}-8^{\circ} \mathrm{C}$ y $4^{\circ} \mathrm{C}-5^{\circ} \mathrm{C}$, respectivamente; en comparación al rango de la media, T: $11^{\circ} \mathrm{C}-12^{\circ} \mathrm{C}$, en las estaciones de Gijón y de Bilbao y de $9^{\circ} \mathrm{C}-10^{\circ} \mathrm{C}$ en la de Logroño. Sin embargo, ese valor porcentual correspondiente al conjunto de jornadas de Viento Sur (VS) solo es superior al correspondiente al conjunto de jornadas otoño-invernales con vientos de las otras direcciones cardinales de viento (VE, $\mathrm{VO}, \mathrm{VN}$ ) en las estaciones de Bilbao especialmente y en la de Logroño [Tabla 7; \%D > rango media $\mathrm{T}(\mathrm{OI})]$.

Las jornadas otoño-invernales de Viento Sur (VS) que en las estaciones de Bilbao, 44\% y Gijón, 37\% registran valores de Temperatura media (T) superiores al rango de su respectiva media otoño-invernal, rango $11^{\circ} \mathrm{C}-12^{\circ} \mathrm{C}$, representan el $66 \%$ y el $51 \%$ del total de días otoño-invernales que en esas estaciones registran valores de Temperatura media superiores al rango de su media correspondiente y estos valores porcentuales son los más altos, especialmente en Bilbao, en relación a los que observan las demás direcciones cardinales de viento (VE, VO, VN) en estas dos estaciones [Tabla 7; \%Tot. $>$ rango media $\mathrm{T}(\mathrm{OI})]$.

\subsection{Humedad relativa media (H) y Viento Sur (VS)}

En las estaciones de Bilbao, 71\%; Gijón, 75\%, así como en la de Vitoria, 73\%, los valores de Humedad relativa media $(\mathrm{H})$ correspondientes al conjunto de jornadas otoñoinvernales con vientos predominantes del Sur o Viento Sur (VS) son inferiores o notablemente inferiores a su correspondiente media otoño-invernal; si bien, las medias de Bilbao, 75\% y Gijón, 76\% son considerablemente inferiores a la media de Vitoria, 79\%. En las estaciones de Burgos ambas medias, otoño-invernal y la correspondiente a las situaciones de Sur (VS) observan el mismo registro, 86\% y en la de Logroño este valor es ligeramente superior al del periodo otoño-invierno, pero esa media otoño-invernal es del 72\%; con notable diferencia, la más baja de entre las cinco estaciones (Tabla 8).

Prácticamente la misma tendencia que para el conjunto de Viento Sur (VS) se observa para el conjunto de jornadas otoño-invernales del sureste (SE), las cuales registran valores de Humedad relativa media $(\mathrm{H})$ inferiores a su media otoño-invernal (OI) en Bilbao y Vitoria especialmente, iguales a ellas en Gijón y Burgos y muy superior a la misma en el caso de Logroño, donde y cuando la influencia de flujos mediterráneos del sureste (SE) es mayor. Únicamente en la estación de Bilbao el registro de Humedad relativa media correspondiente al conjunto de jornadas con vientos predominantes del sureste (SE) es inferior al registro para el conjunto de días otoño-invernales de 
Viento Sur (VS). El conjunto de situaciones del sureste (SE) otoño-invernales registran en las estaciones de Gijón y Logroño el valor de humedad relativa media más alto en comparación a las otras dos direcciones de Sur (S-SO). En la estación de Vitoria los tres registros (SE-S-SO) son iguales

En las cinco estaciones los registros de Humedad relativa media $(\mathrm{H})$ correspondientes a las situaciones otoño-invernales con vientos predominantes de la dirección sur (S) son muy inferiores o inferiores a sus respectivas medias otoño-invernales. Y a su vez son también inferiores, excepto en las estaciones de Gijón y de Vitoria, a la media correspondiente para el conjunto de situaciones de Sur (VS). El registro de Humedad relativa media correspondiente al conjunto de situaciones otoño-invernales con vientos predominantes de la dirección sur (S) es en la estación de Bilbao el más bajo, $68 \%$, tanto para el periodo otoño-invierno (OI), como para el conjunto situaciones de Viento Sur y para cualquiera de las direcciones sectoriales de Sur (SE-S-SO) en cada estación (VS). También, aunque con mucha menos diferencia respecto a sus medias (OI, VS), es el registro más bajo en la estación de Burgos, 85\% (Tabla 8).

Tabla 8. Valores de humedad relativa media (H\%) para el periodo otoño-invierno, conjunto de situaciones otoño-invernales de Viento Sur (VS) y conjunto de jornadas con vientos predominantes de cada una de las direcciones de sur (SE-S-SO), en ese periodo otoño-invierno y en cada una de las estaciones de la región analizadas

\begin{tabular}{|l|c|c|c|c|c|}
\hline \multicolumn{1}{|c|}{ H } & OI & VS & SE & $S$ & SO \\
\hline Bilbao & 75 & 71 & 70 & 68 & 76 \\
\hline Gijón & 76 & 75 & 76 & 75 & 74 \\
\hline Vitoria & 79 & 73 & 73 & 73 & 73 \\
\hline Burgos & 86 & 86 & 86 & 85 & 88 \\
\hline Logroño & 72 & 73 & 79 & 71 & $\mathbf{6 9}$ \\
\hline
\end{tabular}

Por el contrario, son las situaciones otoño-invernales con vientos predominantes del suroeste (SO) las que registran los valores más bajos de humedad relativa media $(\mathrm{H})$ en las estaciones de Gijón y de Logroño, tanto para el periodo otoño-invierno (OI) y conjunto de situaciones de Viento Sur (VS) respectivos, como respecto a cualquiera de las otras direcciones sectoriales de Sur (SE-S). Junto al registro de Bilbao (H: 68\%) correspondiente a las situaciones del sector sur (S), en la estación de Logroño el registro correspondiente al conjunto de jornadas otoño-invernales con vientos predominantes del suroeste (SO), 69\%, es el valor más bajo respecto a la media del periodo otoño-inver- 
nal, respecto al conjunto de jornadas de Viento Sur (VS) y respecto a cualquiera de las direcciones sectoriales de Sur (SE-S-SO) en las cinco estaciones analizadas (Tabla 8).

$\mathrm{Y}$, sin embargo, en la estación de Bilbao este registro correspondiente al conjunto de jornadas otoño-invernales del suroeste (SO) es del 76\%, registro superior a su media otoño-invernal, a la media para el conjunto de jornadas de Sur o Viento Sur (VS) y un $8 \%$ superior al registro correspondiente al conjunto de situaciones del sector sur (S) (Tabla 10).

En las estaciones de Bilbao, Gijón y Vitoria los registros de Humedad relativa media $(\mathrm{H})$ correspondientes al conjunto de jornadas otoño-invernales de Sur o de Viento Sur (VS) son inferiores a los registros correspondientes al conjunto de jornadas otoñoinvernales para cada una de las demás direcciones cardinales de viento; VE, VO, VN; si bien, en la estación de Gijón este valor de Humedad relativa media correspondiente al conjunto de jornadas otoño-invernales del Sur (VS) y el correspondiente a las jornadas del Oeste (VO) son iguales. Los registros de Humedad relativa media $(\mathrm{H})$ de las jornadas otoño-invernales de Sur o de Viento Sur (VS) en las estaciones de Bilbao y Vitoria son notablemente más bajos, $71 \%$ y $73 \%$ respectivamente, que el correspondiente al resto de direcciones cardinales o principales de viento en esas estaciones (Tabla 9).

Tabla 9. Valores de humedad relativa media $(H)$ otoño-invernal correspondientes a cada dirección cardinal de viento (VE, VS, VO, VN), en cada estación analizada

\begin{tabular}{|c|c|c|c|c|c|}
\hline $\mathbf{H}$ & $\mathbf{B i}$ & $\mathrm{Gi}$ & $\mathrm{Vi}$ & $\mathrm{Bu}$ & Lo \\
\cline { 2 - 6 } & 76 & 77 & 77 & 85 & 77 \\
\hline VE & 71 & 75 & 73 & 86 & $\mathbf{7 3}$ \\
\hline VO & 77 & 75 & 79 & 88 & 69 \\
\hline VN & 79 & 79 & 82 & 86 & 72 \\
\hline
\end{tabular}

En las estaciones de Burgos y Logroño los valores de Humedad relativa media $(\mathrm{H})$ más bajos se corresponden con el conjunto de jornadas otoño-invernales de vientos del Este (VE) en Burgos y del Oeste (VO) en Logroño.

El 73\% en la estación de Vitoria de las jornadas otoño-invernales con vientos predominantes del Sur (SE-S-SO) o Viento Sur (VS) registran valores de Humedad relativa media $(\mathrm{H})$ inferiores al rango que incluye su media otoño-invernal, rango 80\%-100\%. Estos valores porcentuales en el resto de estaciones son muy inferiores; $22 \%$ en Bilbao 
y de tan solo el 7\% y 8\% en las de Gijón y Logroño, si bien sobre un rango para su media, en los tres casos, de 60\%-80\%. En la estación de Burgos, para el mismo rango que el de la media de Vitoria, 80\%-100\%, este valor porcentual es de 15\% (Tabla 10).

Tabla 10. Número de jornadas (\%D) para cada dirección principal o cardinal de viento [vientos del Este (VE); vientos del Sur o Viento Sur (VS); vientos del Oeste (VO) y vientos del Norte o Viento Norte $(\mathrm{VN})$ ] y en cada estación meteorológica de la región analizada, en que se registran valores de humedad relativa media inferiores al rango de la media respectiva para el periodo otoño-invierno [\% $\mathbf{D}$ $<$ rango media $\mathbf{H}(\mathbf{O I})$ ]. Valor porcentual que ese número de jornadas, \%D, representa sobre el total de jornadas o días otoño-invernales en los que se registran valores de humedad relativa media inferiores al rango de la media respectiva para el periodo otoño-invernal [\% Tot. < rango media $\mathbf{H}(\mathbf{O I})$ ]. M (OI): Humedad relativa media periodo otoño-invierno

\begin{tabular}{|c|c|c|c|c|c|c|c|c|c|c|c|}
\hline & \multicolumn{5}{|c|}{$<$ rango media $\mathrm{H}(\mathrm{OI})$} & & \multicolumn{5}{|c|}{$<$ rango media $\mathrm{H}(\mathrm{OI})$} \\
\hline$\% \mathrm{D}$ & $\mathrm{Bi}$ & Gi & Vi & $\mathrm{Bu}$ & Lo & $\%$ Tot & $\mathrm{Bi}$ & Gi & Vi & $\mathrm{Bu}$ & Lo \\
\hline VE & 13 & 5 & 61 & 24 & 6 & VE & 40 & 42 & 9 & 54 & 11 \\
\hline VS & 22 & 7 & 74 & 15 & 8 & VS & 86 & 59 & 40 & 27 & 13 \\
\hline VO & 7 & 8 & 59 & 11 & 19 & VO & 19 & 36 & 46 & 16 & 78 \\
\hline VN & 2 & 1 & 42 & 23 & 18 & $\mathbf{V N}$ & 4 & 7 & 33 & 28 & 55 \\
\hline$M(O I)$ & 75 & 76 & 79 & 86 & 72 & $M(O I)$ & 75 & 76 & 79 & 86 & 72 \\
\hline
\end{tabular}

Y es en la estación de Bilbao, especialmente, y en la de Vitoria, en las que estos valores porcentuales de jornadas otoño-invernales de Viento Sur (VS) con registros he Humedad relativa media inferiores al rango en que se ubica la media del periodo otoñoinvierno correspondiente son superiores o muy superiores a los valores porcentuales correspondientes al resto de direcciones cardinales de viento (VE, VO, VN) (Tabla 10; $\% \mathrm{D}<$ rango media $\mathrm{H}(\mathrm{OI})]$. 
Sin embargo en todas las estaciones se generen con mayor o menor intensidad estos procesos de transformación termodinámica e higrométrica que caracterizan a los vientos de montaña especialmente a las advecciones de Sur o situaciones de Viento Sur (VS), las cuales en su conjunto se corresponden con Temperaturas medias (T) cuando menos iguales a las de su media otoño-invernal correspondiente o superiores y observan altos o muy altos valores porcentuales de frecuencia en las que se registran valores superiores de Temperatura media al del rango en que se sitúa su media otoñoinvernal respectiva (Tabla 11).

Ahora bien, únicamente en las estaciones situadas a sotavento del sistema Cántabro-Pirenaico Occidental (Gijón y Bilbao) las situaciones o jornadas de Sur (VS) en que se registran valores de Temperatura media $(\mathrm{T})$ superior a la media del periodo otoñoinvernal respectivo suponen, incluso notablemente en el caso de Bilbao, más de la mitad del total de jornadas o días del periodo otoño-invierno con registros superiores al de la Temperatura media para ese semestre otoño-invernal,

Al igual que observamos para el parámetro de temperatura media (T), destaca el que, para una media otoño invernal de Humedad relativa media $(\mathrm{H})$ notablemente baja, como la de Bilbao, casi la cuarta parte de la situaciones o jornadas de sur (VS) registren valores de humedad relativa media $(H)$ inferiores al rango en que se sitúa su media otoño-invernal (60\%-80\%). Y mucho más alto es ese valor porcentual para la estación de Vitoria, pero para un rango de la media de 80\%-100\% (Tabla 11).

E igualmente, también, a lo que los datos temperatura media (T) nos aportan, son las dos estaciones más septentrionales, a sotavento de sistema Cántabro-Pirenaico Occidental, y muy especialmente la de Bilbao, en las que las jornadas de Sur (VS) registran valores de Humedad relativa media $(\mathrm{H})$ inferiores al rango de su media otoño-invernal representan casi 9 de cada de cada diez jornadas otoño-invernales en la que se registran valores inferiores al rango de esa media otoño-invernal y casi 6 de cada diez en la estación de Gijón. Y destaca también, en otra medida, la diferencia para esos valores porcentuales entre las estaciones de Burgos y Logroño respecto a la de Vitoria. 
产

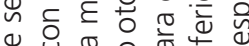
용 은 응

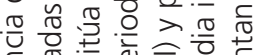

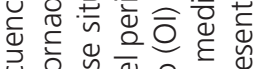

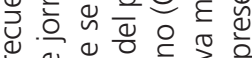

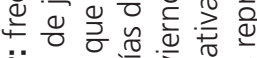

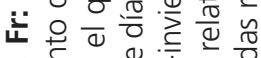
o.

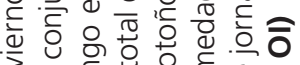

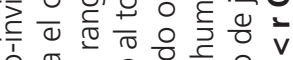

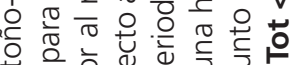

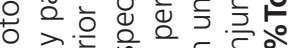

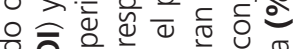
으의 은

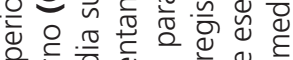

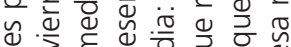

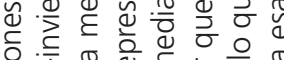

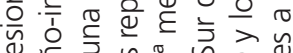

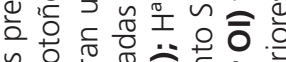

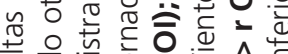

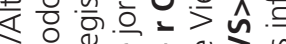

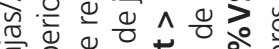

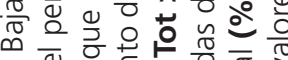

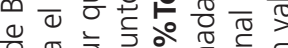

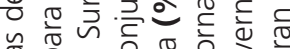

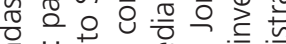
ซ

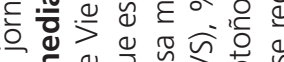

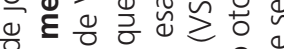

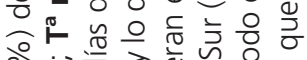
o

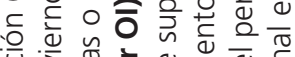

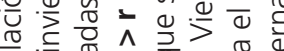

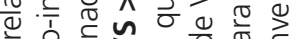

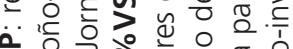
i⿱ t) 范 河 은

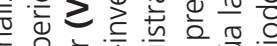

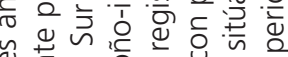
凹ัँ

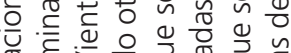

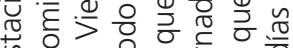
山 응 응 드웡

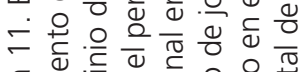

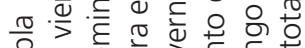

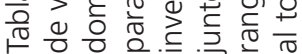

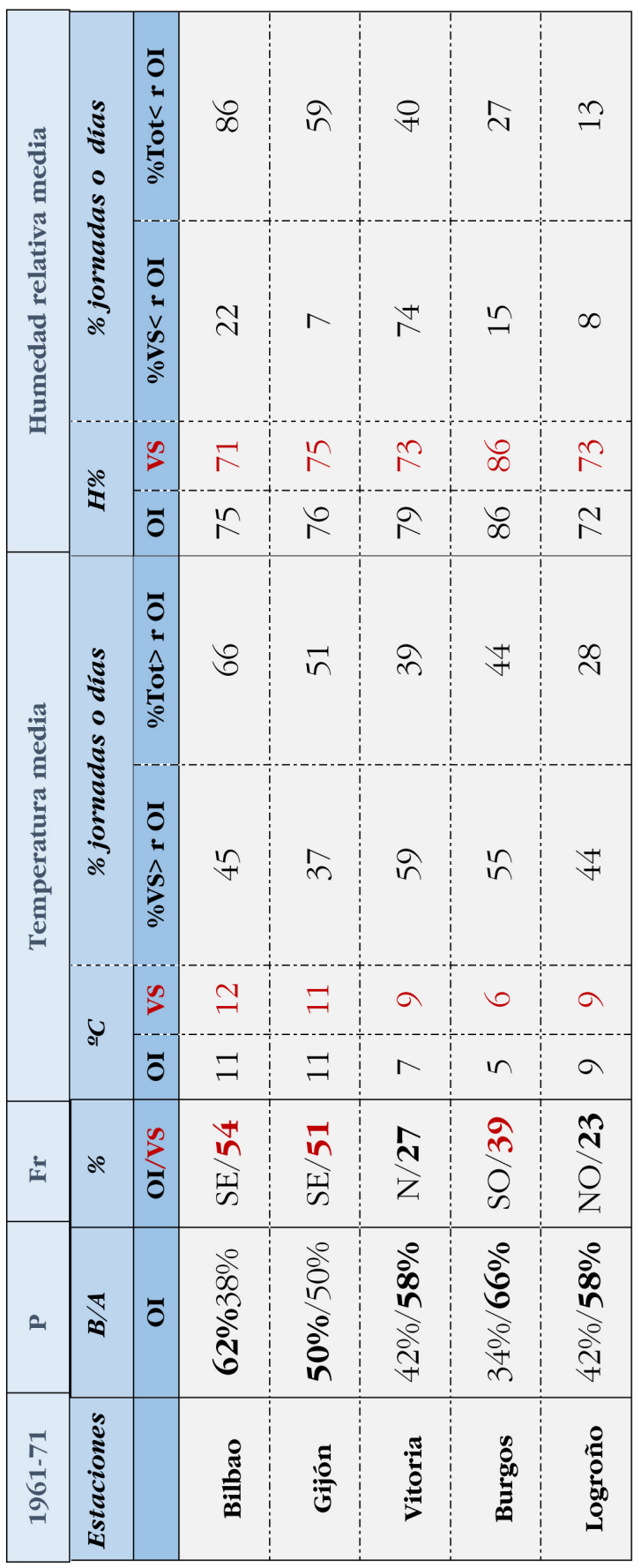




\section{Discusión}

Por lo general los estudios sobre la transformación física e influencia climática de los vientos de montaña y en concreto también en lo referente al Viento Sur sobre la zona septentrional de la Península Ibérica, se suelen centran en el conjunto de esa amplia región, al estar separada del interior peninsular por el abrupto complejo montañosos que configura la extensa Cordillera Cantábrica. Y si bien estos procesos quedan bien definidos en cuanto a los más destacados sistemas orográficos, existiendo infinidad de estudios sobre el efecto de los mismos en la Cordillera Alpina, Cárpatos, Rocosas, etc. (Honka, 1982 y 1985; Gerbier y Berenguer, 1961); sin duda, estos procesos se observan igualmente y con un alto grado de influencia climática también ante otras configuraciones orográficas, aunque estas sean de "escasa elevación", como es el caso de los Montes Vascos y el Pirineo Atlántico, máxime ante advecciones oceánicas e inestables que impulsan vientos templados y húmedos del Sur (SE-S-SO). La escasa elevación no es un factor determinante para que se generen procesos notables de tipo adiabático e incluso de tipo Föhn, cuando un flujo de viento encara un sistema orográfico; y, sin embargo lo que mayor determinación dinámica y atmosférica supone es la extensión, la prolongación, la masificación ininterrumpida de esos sistemas orográficos que los flujos perpendiculares de viento no tienen capacidad de bordear y se ven obligados a superar.

Así, en nuestro estudio evidenciamos que tanto la frecuencia como la influencia de estos flujos de Sur, afectados por fuertes procesos adiabáticos (termodinámicos e higrométricos) como consecuencia del denominado efecto Föhn o Foehn, sobre los registros de otras variables atmosféricas y parámetros climáticos (presión, temperatura media, humedad relativa media, velocidad del viento...), muy especialmente en el periodo otoño-invernal (octubre-diciembre-marzo), quedan definidas por sólidos datos obtenidos a partir de registros diarios de un conjunto de estaciones situadas en la zona oriental de esa franja septentrional peninsular y que se extiende sobre ambas vertientes del continuo orográfico Cordillera Cantábrica Oriental-Montes Vascos-Pirineos Atlánticos.

La influencia climática atemperadora y de reducción muy notable de los valores registrados de humedad relativa generada por estas advecciones de Sur o flujos de Viento Sur se prolonga, como ha sido bien estudiado, no solo por el resto de la zona oriental cantábrica, sino muy probablemente (hipótesis en estudio actualmente), a través de la moderada barrera orográfica Pirenaica occidental, afectando a zonas de la Nueva Aquitania francesa, en lo que conjuntamente hemos venido a denominar como región Cántabro-Pirenaica Occidental, la cual se estructura en torno al eje central del Golfo de Vizcaya-Gascuña.

Y es así, porque esa región Cántabro-Pirenaica Occidental, se sitúa en la trayectoria natural y propia de las borrascas y frentes atlánticos, así como de las advecciones o flujos mediterráneos a través de la Cuenca del Ebro y de los flujos que circulan por la depresión generada entre la vertiente norte pirenaica, la Montaña Negra y el Macizo 
Central francés (Vientos de Autan) y cuya influencia va más allá de lo que se suponía (Hervé, M. y Zambeaux, A.; 2002) alcanzando, con un nivel de afección diferenciado, las regiones de Landas y especialmente Pirineos Atlánticos.

\section{Conclusiones}

El estudio de la influencia del Viento Sur sobre la región Cántabro-Pirenaica Occidental, basándonos en el periodo decenal 1961-1971, serie en la que tanto el valor del índice NAO para el total de los once años, siendo la única serie que observa un valor negativo entre las décadas de 1960 a 2000, como sobre todo por su número de índices anuales negativos (8) que registra la misma, nos aporta datos y conclusiones muy significativas de la relación entre el comportamiento del patrón de presión oceánica NAO y el modelo de circulación atmosférica que genera, junto a otros factores y patrones, sobre la zona suroccidental europea y sobre la Península Ibérica, proyectando advecciones y flujos del Sur, o de Viento Sur, de gran trascendencia climática en la franja septentrional peninsular (con prolongación continental) y especialmente sobre la áreas geográficas situadas en la vertiente a sotavento del sistema orográfico Cántabro-Pirenaico Occidental y en el entorno geográfico territorial que se sitúa a ambos flancos el eje central del Golfo de Vizcaya.

\section{Agradecimientos}

A AEMET por proporcionar los datos en los que se basa este artículo. A Askoa Ibisate y Ana Sáenz de Olazagoitia, profesoras de Geografía Física de la UPV/EHU, por la revisión, aportaciones al manuscrito y colaboración imprescindible en el diseño de los mapas.

\section{Bibliografía}

Autan (Antane, Autune); Weather Online; DOI: https://www.woespana.es/reports/wind/The-Autan.htm (última consulta enero 2021).

Bove, M.C., Elsner, J.B., Landsea, C.W., Niu, X., and J.J. O'Brien (1998). Effects of El Niño on U.S. landfalling hurricanes, revisited. Bulletin of the American Meteorological Society, 79, pp. 2477-2482.

ECA\&D (European Climate Assessment \& Dataset); DOI: https://www.eea.europa.eu/data-andmaps/indicators/global-and-european-temperature-9/european-climate-assessment-datasetproject 
El Viento Sur o Efecto Föehn; EL Viento Sur en Comillas; MeteoComillas; DOI: http://www.meteocomillas.com/index.php?option=com_wrapper\&view=wapper\&Itmited=116 (Última consulta: 20/03/21 a las 09:34 h).

Fernández García, F. y Rasilla, D. (1992). El viento en la Cornisa Cantábrica: Avance de un estudio sobre los temporales de S. Cuadernos de Sección. Historia 20, pp. 271-295. Donostia: Eusko Ikaskuntza.

Gerbier, N. and Berenger, M. (1961). Experimental studies of lee waves in the French Alps. Quart, J.R.; Met. Soc. 87, pp. 13-23.

Guarin, D. y Taboada, J.J. (2010). Influencia de los diferentes patrones de teleconexión en el Atlántico Norte sobre la lluvia y precipitación en Galicia (NO España). Guarin y Taboada, ACT 1, pp. 81-94.

Hoinka, K.P. (1985). Observación del flujo de aire sobre los Alpes durante un evento de Foehn. DOI: https://doi.org/10.1002/qj.49711146709.

Hurrell, J.W. (1995). Decadal Trends in the North Atlantic Oscillation: Regional Temperatures and Precipitation. Science, 269 (5224): pp. 676-679. DOI: 10.1126/science.269.5224.676.

Jiménez Quiroz, Ma del C. (2020). Indicadores Climáticos. Una manera para identificar la variabilidad climática a escala global. Anexo del Informe Técnico: Elaboración de un boletín con información hidroclimática de los mares de México. INAPESCA (Instituto Nacional de Pesca, Gobierno de Mexico), Boletines, Hidroclimático, 32(11), pp. 497-50. DOI: https://www.inapesca.gob.mx/portal/documentos/publicaciones/Boletines/hidroclimatico/Indices-climaticos.pdf.

La Haute-Garonne, enciclopedia ilustrada, Hervé Martin y Alain Zambeaux, ediciones Privat, septiembre de 2002 (ISBN 2-7089-5811-9), página 120, Le vent d'autan

Ledesma, M. (1959). Ondas de montaña sobre España Central. Condiciones sinópticas que producen ondas de sotavento causadas por vientos del sur sobre el sistema montañoso central de España. Ministerio del Aire. Publicaciones, Serie A (Memorias), nº 33. Madrid.

McKnight, T.L. \& Hess, D. (2000). Katabatic Winds. En, Physical Geography: A Landscape Appreciation, pp. 131-2. Upper Saddle River, NJ: Prentice Hall.

National Weather Service-Climate Prediction Centre; NOAA-CPC; DOI: https://origin.cpc.ncep. noaa.gov/products/analysis_monitoring/ensostuff/ONI_v5.Php; última consulta febrero 2021.

NOAA-CPC: El Niño-Southern Oscillation (ENSO). DOI: https://www.cpc.ncep.noaa.gov/products/precip/CWlink/MJO/enso.shtml; última consulta enero 2021.

Pascual Berghaenel, R. (2002). Meteorología de montaña; $R A M$, artículo recuperado por Francisco Martín, oct 2013; DOI: https://studylib.es/doc/6269280/meteorolog\%C3\%ADa-de-monta\% C3\%B1a-introducci\%C3\%B3n

Rasilla, D.F. (1990). Viento del sur y efecto Föhn en la vertiente septentrional de la Cordillera Cantábrica. XIV Encuentro de Jóvenes Geógrafos, Zaragoza, pp. 129-140.

Thillet, J.J. (1998). La meteorología de montaña. Barcelona: Martínez Roca.

Ugarte, F.M. (1982). Observaciones sobre el efecto Föehn en el pie de monte de la Sierra de Aizkorri-Aloña (S. de Guipúzcoa). Munibe Sociedad de Ciencias Aranzadi. San Sebastián Vol. 34, n⿳⺈ 4, pp. 289-294. 
Uriarte, A. (1985). El viento en la costa del País Vasco. Cuadernos de Sección. Historia Geografía /Sociedad de Estudios Vascos. San Sebastián, T. 5. pp. 55-74.

Uriarte, A. (1996). El clima. En Ruiz Urrestarazu, E. y Meaza, G. (eds.) Geografia de Euskal Herria. Tomo 3. Clima y aguas. ETOR, pp. 6-83, Donostia.

Viers, G. (1964). Progrés dans le connaissance du foehn. Revue de Geographie des Pyrenees et du ud Oest, 42, p. 414

Woollings, T., Franzke, C., Hodson, D.L.R., Dong, B., Barnes, E.A., Raible, C.C. and Pinto, J.G. Contrasting interannual and multidecadal NAO variability. United States: N. p., 2014. Web. DOI: 10.1007/s00382-014-2237-y.

Zúñiga López, I. y Crespo del Arco, E. (2010). Meteorología y Climatología; Editorial UNED, $2^{\text {a }}$ ed., $1^{\mathfrak{a}}$ imp. (04/2010), p. 263. 